\title{
Association Between ZNF804A Gene rs 1344706 Polymorphism and Brain Functions in Healthy Individuals: A Systematic Review and Voxel-Based Meta-Analysis
}

\author{
Liqiong Yang' \\ Fan $X^{2}$ \\ $\mathrm{Yi} \mathrm{He}^{3}$ \\ Yanzhang $\mathrm{Li}^{4}$ \\ Zi Chen ${ }^{4}$ \\ Shuai Wang ${ }^{4}$ \\ 'Department of Pharmacy, Chengdu \\ Medical College, Chengdu, 610500, \\ People's Republic of China; ${ }^{2}$ Department \\ of Public Health, Chengdu Medical \\ College, Chengdu, 610500, People's \\ Republic of China; ${ }^{3}$ Department of \\ Medicine, National Engineering and \\ Research Center for Natural Medicines, \\ Chengdu, 610400, People's Republic of \\ China; ${ }^{4}$ Department of Psychology, \\ Chengdu Medical College, Chengdu, \\ 610500, People's Republic of China
}

\begin{abstract}
Objective: Zinc finger protein 804A (ZNF804A) protein participates in embryonic neural repair and development. The single nucleotide polymorphism rs1344706 in ZNF804A gene is closely related to functional abnormalities of the human brain. However, these results are inconsistent. This association was verified by meta-analysis in this study.

Methods: Fifteen studies on functional magnetic resonance imaging involving 1710 healthy individuals were included in the systematic review and meta-analysis used by Anisotropic Effect-Size Signed Differential Mapping software.

Results: Functional connectivity of the right dorsolateral prefrontal cortex (rDLPFC)-left hippocampus in the rs1344706 risk allele carrier was significantly increased $(z=2.066, p<$ $0.001)$, while those in the rDLPFC-left middle frontal gyrus $(z=-1.420, p<0.001)$ and rDLPFC-right middle frontal gyrus $(z=-1.298, p<0.001)$ were significantly decreased. Neural activity of the left anterior cingulate gyrus in the rs1344706 risk allele carrier was significantly decreased $(z=-2.525, p<0.001)$. Sensitivity analysis was almost stable, and no publication bias was found.

Conclusion: The changes in brain function have a clear correlation with ZNF804A gene in healthy individuals, which indicate the contribution of genetic variants on brain dysfunction.

Registration Number: This meta-analysis is registered in PROSPERO (No. CRD42016051331).
\end{abstract}

Keywords: functional magnetic resonance imaging, zinc finger protein 804A, meta-analysis

\section{Introduction}

Genome-wide association (GWA) studies have revealed that zinc finger protein 804 (ZNF804A) gene is associated with the susceptibility of mental disorders, such as schizophrenia, bipolar disorder, and depression. ${ }^{1-3}$ ZNF804A encodes a transcription factor; it is highly expressed in the brain and involved in the development and functionality of the human brain. ${ }^{4}$ Carriers with risk allele in ZNF804A have higher risk for developing mental illnesses than non-carriers. ${ }^{3}$ A single-nucleotide polymorphism (SNP) named rs1344706 in ZNF804A was first identified by a previous GWA study in worldwide population. ${ }^{5}$ From the studies that detected the functional effect of ZNF804A gene polymorphisms, the most reproducible result is that the risk allele $\mathrm{A}$ in rs1344706 is associated with the neurophenotype of mental disorders, patients' performance, and their treatment prognosis. ${ }^{6,7}$ Our previous meta-analysis in
Department of Psychology, Chengdu Medical College, No. 783 Xindu Avenue, Chengdu, 610500, People's Republic of China

Tel +862862739710

Email wshuai86@I26.com 
Chinese population detected the relationship between rs1344706 genotype distribution and susceptibility and drug efficacy for schizophrenia and concluded that risk allele A carriers in this SNP had higher risk for schizophrenia but not for treatment efficacy. ${ }^{8}$ Furthermore, another meta-analysis that included studies in worldwide population demonstrated a very strong association between the risk allele A in rs1344706 and risk for schizophrenia. ${ }^{9}$

The human brain is the foundation of mental activity. Brain injury and developmental abnormalities can directly lead to the occurrence of mental disorders. ${ }^{10}$ Neuroimaging studies suggest that patients with mental disorders, such as schizophrenia, have certain abnormalities on brain function. "11,12 "Imaging genetics" aims to detect the associations between risk genes and the changes in brain function and/or structure of individuals with mental disorders, and to clarify the contributions of these risk genes to abnormalities in the development of the human brain. Numerous studies showed that the ZNF804A gene might be associated with brain function and structure in healthy individuals and patients with mental disorders. ${ }^{13,14}$ Lencz et al verified the effects of rs1344706 in schizophrenia risk allele gene ZNF804A on neuroanatomy and neurocognitive phenotype. ${ }^{15}$ Research on functional connectivity by Esslinger et al found that the risk allele in rs 1344706 carrier had a significantly increased functional coupling in the right amygdala. ${ }^{16}$ However, another study revealed that risk allele in rs 1344706 had a negative effect on functional connectivity between the right dorsolateral prefrontal cortex (rDLPFC) and the left hippocampus. ${ }^{17}$ For neural activity studies in healthy individuals, risk allele carriers exhibited a significant risk allele dose effect on neural activity in the medial prefrontal cortex and left temporoparietal cortex under a theory-of-mind task. ${ }^{18}$ In a neural activity research based on the amplitude of lowfrequency fluctuation, the risk allele A exhibited a negative effect on the left calcarine gyrus. ${ }^{19}$

Several studies have explored the effects of risk allele in rs1344706 of ZNF804A gene on human brain functional connectivity and neural activity in healthy individuals. However, the affected brain regions and effect sizes are inconsistent due to the differences in clinical baselines and research methods. Previous brain imaging studies also have a relatively small sample size and low statistical test power. In addition, systematic conclusions to comprehensively determine the effects of risk allele $\mathrm{A}$ on brain function are lacking. Therefore, this study aimed to explore these effects by signed differential mapping meta- analysis and systematic review in healthy individuals, which could further determine the important role of the SNP rs1344706 in ZNF804A gene in the development of schizophrenia.

\section{Materials and Methods}

\section{Registration}

This systematic review and meta-analysis was registered in PROSPERO (CRD42016051331).

\section{Study Design}

Voxel-based and general meta-analysis methods based on clinical data of statistical maps, peak coordinates, and statistical effect size, which were collected from previous association studies of brain function changes and ZNF804A gene rs1344706 polymorphism, were used to design the study.

\section{Search Strategy}

This study was conducted in accordance with the Preferred Reporting Items for Systematic Reviews and MetaAnalysis 2020 statement. $^{20}$ PubMed, Medline, ScienceDirect, and Scopus were used to search for literature from January 2006 until April 2021. The following search terms were combined and used: "functional MRI/ $\mathrm{fMRI} /$ functional magnetic resonance imaging/brain function" and "ZNF804A/rs1344706." Publications from conferences, monographs, theses, or reference lists in identified studies were also regarded as potential sources to be included in the systematic review.

\section{Selection Criteria}

The studies that met the following criteria were included in the meta-analysis: 1) original cross-sectional research that detected the association between ZNF804A gene and brain function; 2) individuals scanned by functional magnetic resonance imaging (fMRI) in the whole brain; 3) comparison of brain function among different genotypes in rs1344706 polymorphism; 4) English publication in peer-reviewed journals and monographs. Studies were excluded if they met the following criteria: 1) data were unavailable or generated from region of interest approaches; 2) repetitive publications; 3) conference papers; 4) fMRI method was not used for detection. Two researchers (LY and FX) independently performed the study search and selection. Furthermore, the citations in 
the included studies were searched to identify potential eligible studies.

\section{Data Extraction and Literature Quality Assessment}

Two independent reviewers (LY and SW) extracted the data. In the first screening, all eligible titles and abstracts of studies were carefully reviewed using Endnote software (EndNote X8, Thomson Corporation). The full texts of the remaining papers were evaluated for inclusion in the metaanalysis. Disagreements were resolved through discussion. The two reviewers independently extracted the specific information in the included articles and recorded these in detail in pre-set standard electronic form. The extracted contents were not limited to the following: 1) general information of the studies (publication year and last name of the first author); 2) characteristics of the investigated sample (sample cohort, gender, mean age, size); 3) scanning parameter, including but not limited to the full width at half maximum; 4) distribution of genotypes and alleles of rs1344706; 5) effect size and its peak coordinates of voxel-based data in brain function of statistically significant differences. If the incomplete data could not be addressed by obtaining the rest of the data from the original authors, then the study was excluded. All data were checked for internal consistency. The quality of the included studies was assessed following the checklist that focused on the clinical and demographic aspects of individual study samples and imaging-specific methodology. ${ }^{21}$

\section{AES-SDM Analysis}

Anisotropic effect-size version of seed-based $d$ mapping (AES-SDM) is a meta-analytic method for voxel-based imaging studies. It reports the peak coordinates of gray and/or white matter differences in the whole brain. The user instructions have been described in a previous study. ${ }^{22}$ Several meta-analyses on neuropsychiatric disorders were conducted by AES-SDM, including our two previous works. ${ }^{21,23}$ Four researchers (FX, YH, YL, and ZC) performed the meta-analysis. The main threshold was set at uncorrected $p<0.001$ (empirically equivalent to corrected $p<0.05$ ) with cluster extent $\geq 20$ voxels and $z$ score $>1 .^{21}$ $Q$ statistic value with a threshold of $p<0.05$ was considered to be significantly heterogeneous. ${ }^{24}$ A leave-one-out jackknife method was used in the sensitivity analysis. Funnel plots were then obtained through Egger's test to evaluate the possibility of publication bias. ${ }^{25}$

\section{Results}

\section{General Characteristics of the Included Studies}

A flowchart of the search process is presented in Figure 1. PRISMA checklist of this meta-analysis is presented in the $\underline{\text { supplement material. Fifteen studies }}{ }^{16-19,26-36}$ on brain functional connectivity and activity involving 1710 healthy individuals were included in the systematic review and meta-analysis. The general characteristics and research quality assessment of the included studies are shown in Table 1. The main results of risk allele affected regions in healthy individuals in each study are summarized in Table 2. The different brain regions and different effects of rs1344706 risk allele A on functional connectivity and neural activity were exhibited.

\section{Effect of ZNF804A Gene rs I344706 Risk Allele on Brain Functional Connectivity}

The seed regions reported in 12 studies ${ }^{16-18,26,27,30-36}$ regarding functional connectivity are summarized in Figure 2. Due to the high frequencies of rDLPFC-based seed (reported by eight studies ${ }^{16-18,26,27,30,32,36}$ ), the subsequent AES-SDM analysis of brain functional connectivity mainly focused on the rDLPFC seed. In the healthy population, the functional connectivity of the rDLPFC-left hippocampus in the rs 1344706 risk allele carrier was significantly increased $(z=2.066, p<0.001)$, while those in the rDLPFCleft middle frontal gyrus $(z=-1.420, p<0.001)$ and rDLPFCright middle frontal gyrus $(z=-1.298, p<0.001)$ were significantly reduced (Table 3 and Figure 3 ). Result of connectivity coupling between rDLPFC and left middle frontal gyrus had a significant heterogeneity $(p<0.05)$. The area of interest with peak coordinate only reported in one study was not included in AES-SDM meta-analysis. Generally, the risk allele of rs 1344706 showed mainly negative effects on functional connectivity (Table 4).

\section{Effect of ZNF804A Gene rs I344706 Risk Allele on Neural Activity}

Seven studies ${ }^{18,19,27-30,34}$ reported significant results in neural activity. However, only five studies ${ }^{18,19,27-29}$ had peak coordinates. AES-SDM analyzed the effect of ZNF804A gene rs1344706 risk allele on neural activity. After data integration and image reconstruction, the neural activity of the left anterior cingulate gyrus was significantly decreased in the rs 1344706 risk allele carrier of 


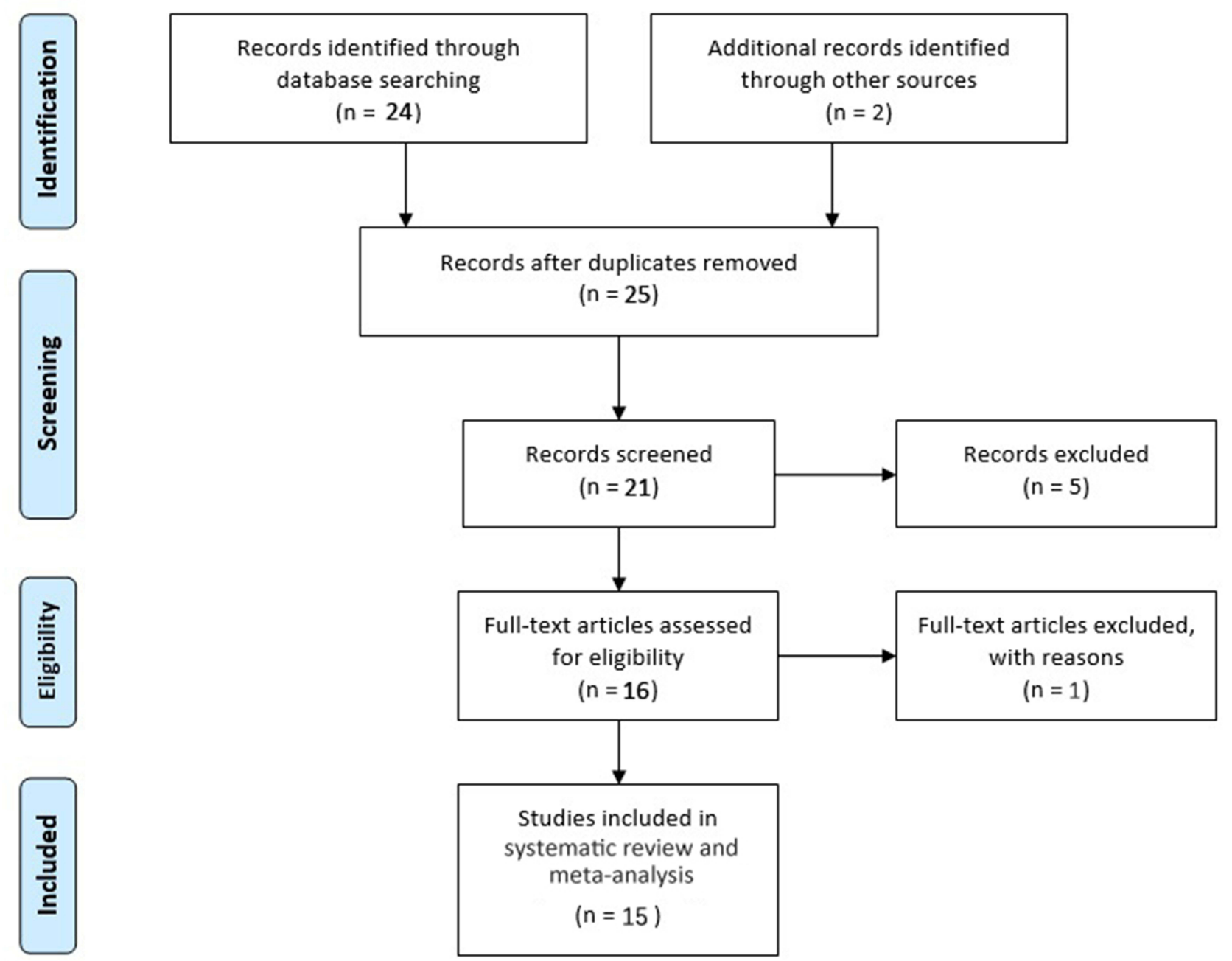

Figure I Flowchart of the selection process.

the healthy population (MNI: $\mathrm{x}=0, \mathrm{y}=34, \mathrm{z}=22 ; z=-2.525$, $p<0.001$; voxels $=2463$ ), and no other brain area showed significantly increased neural activity (Figure 4 ). Result of this brain region had no heterogeneity $(p>0.05)$. The remaining two studies ${ }^{30,34}$ had a negative result of neural activity or did not report the peak coordinates in healthy individuals (Table 4).

\section{Sensitivity Analysis and Publication Bias}

Leave-one-out jackknife analyses showed that most functional connectivity and neural activity results were not significantly changed. When removed the study of Esslinger, ${ }^{16}$ Rasetti, ${ }^{17}$ or Thurin, ${ }^{27}$ respectively, the results were slightly changed (Table 5). Further sensitivity analysis excluded the studies of Esslinger ${ }^{26}$ and Zhao ${ }^{36}$ due to the potential partially duplicated sample. However, the results were not significantly changed (Table 6).
In addition, according to Egger's test, no publication bias was found in functional connectivity and neural activity analyses $(t=1.410, p=0.208 ; t=0.05, p=0.963$; Figure 5$)$.

\section{Discussion}

In this study, AES-SDM was used in the meta-analysis of studies regarding the effects of risk allele in rs1344706 polymorphisms of ZNF804A gene on brain function. The AES-SDM analysis found that in healthy populations, the functional connectivity of the rDLPFC-left hippocampus in the rs1344706 risk allele carrier was significantly increased, but those of the rDLPFC-right middle frontal gyrus and rDLPFC-left middle frontal gyrus were significantly decreased. Analysis of local activity revealed that the neural activity in this risk allele carrier was significantly decreased in the left anterior cingulate gyrus. 


\begin{tabular}{|c|c|c|c|c|c|c|c|c|c|c|c|c|c|c|c|c|}
\hline 胥 & & $\begin{array}{l}\frac{c}{b 0} \\
\frac{.00}{I}\end{array}$ & $\begin{array}{l}\frac{5}{\underline{\underline{a}}} \\
\text { 足 }\end{array}$ & $\begin{array}{l}\text { 点 } \\
\text { 至 }\end{array}$ & $\begin{array}{l}\frac{5}{60} \\
\frac{.00}{I}\end{array}$ & 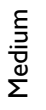 & 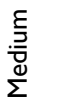 & 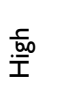 & 喜 & $\frac{\text { 旨0 }}{\text { 竞 }}$ & $\begin{array}{l}\text { 点 } \\
\text { 至 }\end{array}$ & $\begin{array}{l}\frac{c}{\underline{a 0}} \\
\text { 至 }\end{array}$ & $\begin{array}{l}\text { 点0 } \\
\text { 竞 }\end{array}$ & $\begin{array}{l}\text { 品 } \\
\text { 至 }\end{array}$ & $\begin{array}{l}\text { 点 } \\
\text { 至 }\end{array}$ & $\begin{array}{l}\text { 点 } \\
\text { 夏 }\end{array}$ \\
\hline 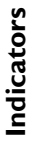 & & U⿺乚 & U⿺乚一 & U & ن & 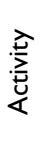 & ن & ن & 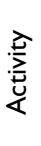 & U⿺乚一 & U & U⿺乚 & ن & U⿺乚 & 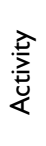 & U \\
\hline 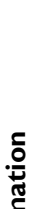 & 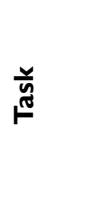 & $\begin{array}{l}\text { v } \\
\text { Ũ } \\
\text { Z }\end{array}$ & 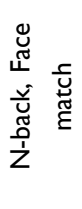 & $\begin{array}{l}\text { 弟 } \\
\text { i் }\end{array}$ & 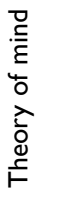 & 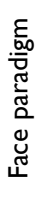 & $\begin{array}{l}\text { v } \\
\text { Ũ } \\
\text { Z } \\
Z\end{array}$ & 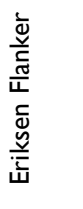 & 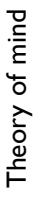 & 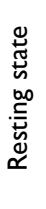 & 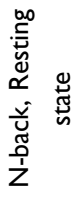 & 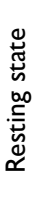 & 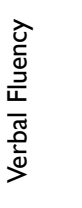 & 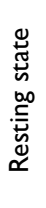 & 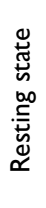 & 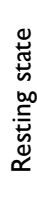 \\
\hline 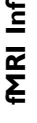 & $\sum_{\amalg}^{\Sigma} E$ & $a$ & $a$ & $\infty$ & $a$ & 乞 & 乙 & $\infty$ & $\sigma$ & 0 & $\infty$ & $\nabla$ & เก & Z & 6 & $\infty$ \\
\hline
\end{tabular}

\begin{tabular}{|c|c|c|c|c|c|c|c|c|c|c|c|c|c|c|c|c|c|}
\hline त्र & 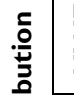 & $\underset{\mathbf{I}}{\mathbf{m}}$ & 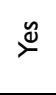 & 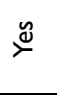 & $\widetilde{\Sigma}$ & $\overbrace{\nu}^{\tilde{y}}$ & 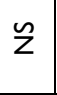 & Z & $\stackrel{\check{\Perp}}{\check{\nu}}$ & $\stackrel{\check{\nu}}{\check{\nu}}$ & $\stackrel{\stackrel{y}{\nu}}{\nu}$ & $\stackrel{\Perp}{\check{\nu}}$ & $\check{\nu}_{\nu}^{\tilde{\nu}}$ & $\stackrel{\check{\nu}}{\sim}$ & $\stackrel{\mathscr{\Xi}}{\simeq}$ & $\stackrel{\check{\varpi}}{\check{\nu}}$ & $\stackrel{\check{\nu}}{\check{\nu}}$ \\
\hline 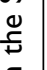 & 䇏 & U & $\bar{\sim}$ & $\bar{\sim}$ & \pm & $\underline{\infty}$ & $=$ & $\bar{\sim}$ & $\stackrel{\sim}{\sim}$ & $\stackrel{\infty}{m}$ & $\stackrel{\sim}{\sim}$ & $\bar{N}$ & $\stackrel{+}{m}$ & $\tilde{z}$ & \pm & $g$ & $\bar{\sim}$ \\
\hline $\begin{array}{l}= \\
0\end{array}$ & 商 & U & in & 요 & ケே & $\dot{q}$ & $\bar{N}$ & +ా & m & $\widehat{\infty}$ & 0 & $\stackrel{m}{q}$ & $\widetilde{\sigma}$ & $\tilde{z}$ & ケ & 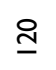 & q \\
\hline & ర๊̃ & $\varangle$ & $\stackrel{m}{\sigma}$ & q & $\hat{m}$ & $\mathcal{F}$ & $=$ & $\hat{\wedge}$ & ळ & ติ & $\stackrel{\sim}{\sim}$ & $\ddot{\sim}$ & $\tilde{m}$ & $\check{z}$ & $\stackrel{\sim}{\sim}$ & $\dot{q}$ & $\ddot{\sim}$ \\
\hline $\overrightarrow{\mathrm{t}}$ & 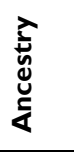 & & 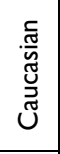 & 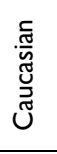 & $\tilde{z}$ & 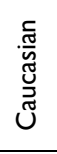 & 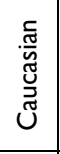 & 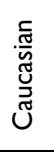 & 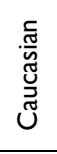 & 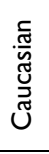 & 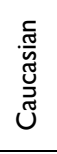 & 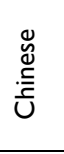 & 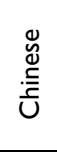 & 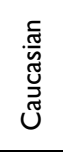 & 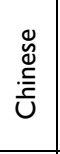 & $\begin{array}{l}\stackrel{\mathscr{U}}{~} \\
\stackrel{\underline{E}}{\bar{U}}\end{array}$ & 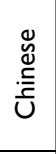 \\
\hline$\lambda$ & $\stackrel{\times}{5}$ & & $q$ & $q$ & F & $\hat{f}$ & \pm & $\stackrel{\circ}{\circ}$ & $\underline{\underline{0}}$ & d & $\ddot{\sim}$ & $\stackrel{\infty}{\infty}$ & $\alpha$ & 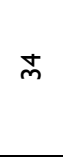 & $\mathscr{g}$ & $\stackrel{\llcorner}{\infty}$ & $\underline{a}$ \\
\hline$\ddot{y}$ & كُ & & $\stackrel{\infty}{\stackrel{m}{m}}$ & $\stackrel{\infty}{\underset{m}{m}}$ & $\stackrel{\stackrel{n}{m}}{n}$ & $\underset{m}{m}$ & 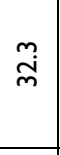 & $\overline{\tilde{\sim}}$ & $\frac{\dot{m}}{m}$ & $\overline{\dot{m}}$ & \pm & 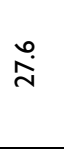 & $\grave{\lambda}$ & $\bar{\rho}$ & $\begin{array}{l}\stackrel{\infty}{\infty} \\
\stackrel{\sim}{n}\end{array}$ & ㅇ. & $\stackrel{\sim}{\sim}$ \\
\hline$\stackrel{n^{0}}{=0}$ & 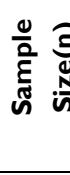 & & $\underline{\underline{\underline{n}}}$ & $\equiv$ & ஃ & oิ & $\mathscr{F}$ & む & : & $\stackrel{\infty}{\stackrel{\infty}{-1}}$ & in & $\hat{\infty}$ & $\stackrel{\infty}{\simeq}$ & ळ & $\alpha$ & $\frac{\infty}{N}$ & $\stackrel{\infty}{ \pm}$ \\
\hline 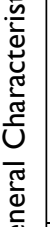 & 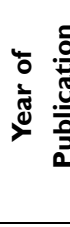 & & ఫ્ণે & $\overline{\bar{N}}$ & $\overline{\bar{i}}$ & $\overline{\bar{i}}$ & $\frac{m}{i}$ & $\frac{m}{i}$ & $\frac{m}{i}$ & $\frac{\nabla}{i}$ & $\stackrel{\frac{n}{2}}{\stackrel{N}{N}}$ & $\stackrel{\circ}{\circ}$ & $\frac{\infty}{i}$ & $\stackrel{\infty}{i}$ & $\stackrel{\infty}{\circ}$ & $\frac{\sigma}{\bar{N}}$ & ્ָరి \\
\hline 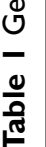 & 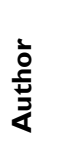 & & 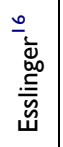 & 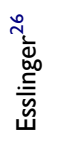 & 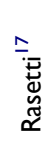 & 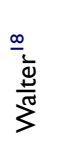 & 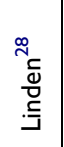 & $\frac{m_{n}}{\frac{m_{\bar{z}}}{\bar{I}}}$ & 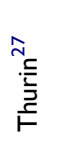 & 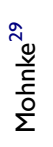 & $\begin{array}{c}\bar{c} \\
: \bar{c} \\
\overline{\bar{y}} \\
\overline{0} \\
u\end{array}$ & $\begin{array}{l}N_{\infty} \\
\text { 步 } \\
\frac{\pi}{N}\end{array}$ & $\begin{array}{l}\stackrel{m}{C}_{\bar{U}} \\
\underset{U}{U}\end{array}$ & 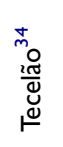 & 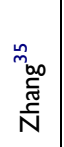 & $\stackrel{\circ}{\bar{u}}$ & $\begin{array}{l}\stackrel{0}{0} \\
\stackrel{0}{N} \\
\frac{\pi}{N}\end{array}$ \\
\hline
\end{tabular}


Table 2 The Main Results of Risk Allele Effected Regions in Healthy Individuals in the Included Studies

\begin{tabular}{|c|c|c|}
\hline Study & Significant Region in Functional Connectivity & $\begin{array}{l}\text { Significant Region in Neural } \\
\text { Activity }\end{array}$ \\
\hline Esslinger $2009^{16}$ & Right amygdala, rDLPFC-HF & - \\
\hline Esslinger $201 \mathrm{I}^{26}$ & $\begin{array}{l}\text { rDLPFC-left middle frontal gyrus, rDLPFC-right middle frontal gyrus, rDLPFC-right } \\
\text { superior frontal gyrus, et al. }\end{array}$ & - \\
\hline Rasetti $2011^{17}$ & rDLPFC-HF, rDLPFC-inferior parietal lobule & - \\
\hline Walter $201 I^{18}$ & $\begin{array}{l}\text { ITPJ-inferior frontal gyrus, ITPJ-cuneus, rDLPFC-middle frontal gyrus, rDLPFC-precentral } \\
\text { gyrus, et al. }\end{array}$ & $\begin{array}{l}\text { DLPFC, DMPFC, VLPFC, TPJ, } \\
\text { IPL, PCC }\end{array}$ \\
\hline Linden $2013^{28}$ & - & Right inferior frontal gyrus \\
\hline Paulus $2013^{30}$ & rDLPFC-left HF, rDLPFC-right HF & No significant region was found \\
\hline Thurin $2013^{27}$ & rDLPFC-anterior cingulate cortex & $\begin{array}{l}\text { rDLPFC, anterior cingulate } \\
\text { cortex }\end{array}$ \\
\hline Mohnke $2014^{29}$ & ITPJ-inferior frontal gyrus & TPJ, DMPFC, PCC \\
\hline Cousijn $2015^{31}$ & Hippocampal-prefrontal cortex & - \\
\hline Zhang $2016^{32}$ & rDLPFC-left HF & - \\
\hline Chen $2018^{33}$ & No significant region was found & - \\
\hline Tecelão $2018^{34}$ & - & Left inferior frontal gyrus \\
\hline Zhang $2018^{35}$ & Left HF-rDLPFC & - \\
\hline Cui $2019^{19}$ & - & Left calcarine gyrus \\
\hline Zhao $2020^{36}$ & rDLPFC-left HF & - \\
\hline
\end{tabular}

Abbreviations: DLPFC, dorsolateral prefrontal cortex; DMPFC, dorsomedial prefrontal cortex; VLPFC, ventrolateral prefrontal cortex; IPL, inferior parietal lobe; PCC, posterior cingulate cortex; TPJ, temporo-parietal junction; HF, hippocampus formation.

The outcomes of the present meta-analysis are generally consistent with previous observational studies, although some differences were observed. The

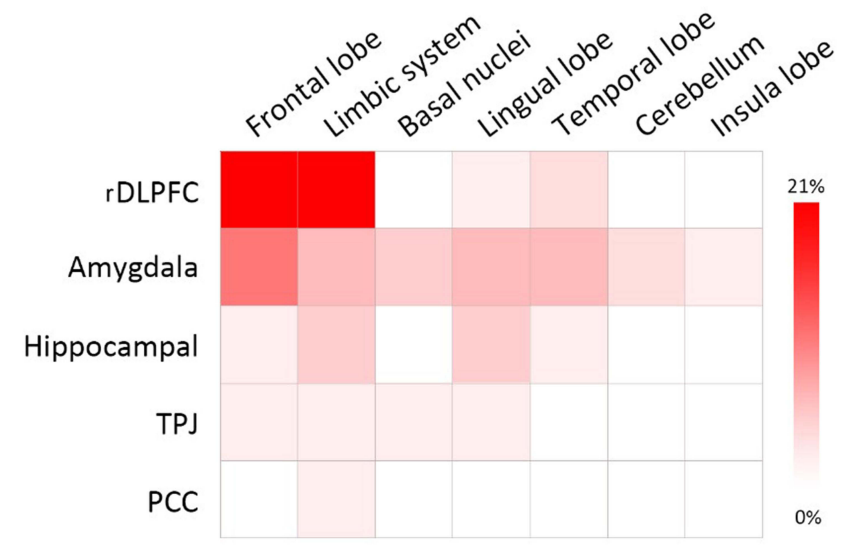

Figure 2 Frequency distribution of brain regions involved in brain functional connectivity.

Abbreviations: rDLPFC, right dorsolateral prefrontal cortex; TPJ, temporoparietal junction; PCC, posterior cingulate cortex.
Table 3 Effect of Rs 344706 Risk Allele on Brain Functional Connectivity Based on rDLPFC Seed Region

\begin{tabular}{|c|c|c|c|c|c|c|}
\hline \multirow[t]{2}{*}{ Connected Region } & \multicolumn{5}{|c|}{ Peak Coordinate } & \multirow{2}{*}{$\begin{array}{l}\text { Cluster } \\
\text { Size in } \\
\text { Voxels }\end{array}$} \\
\hline & \multicolumn{3}{|c|}{ MNI $(x, y, z)$} & SDM-z & $p$ & \\
\hline \multicolumn{7}{|c|}{ Positive effects of rs 1344706 risk alleles } \\
\hline Left hippocampus & -32 & -24 & -12 & 2.066 & $<0.001$ & 888 \\
\hline \multicolumn{7}{|c|}{ Negative effects of rs I 344706 risk alleles } \\
\hline $\begin{array}{l}\text { Left middle frontal } \\
\text { gyrus }\end{array}$ & -46 & 32 & 34 & -1.420 & $<0.001$ & 226 \\
\hline $\begin{array}{l}\text { Right middle } \\
\text { frontal gyrus }\end{array}$ & 30 & 50 & 32 & -1.298 & $<0.001$ & 150 \\
\hline
\end{tabular}

Abbreviation: rDLPFC, right dorsolateral prefrontal cortex.

connectivity couplings such as rDLPFC-inferior parietal lobule, rDLPFC-anterior cingulate cortex, and rDLPFCprecentral gyrus was reported by other studies. ${ }^{18,26,27}$ However, they were not significant in our meta-analysis. Furthermore, we found that risk allele A was associated 


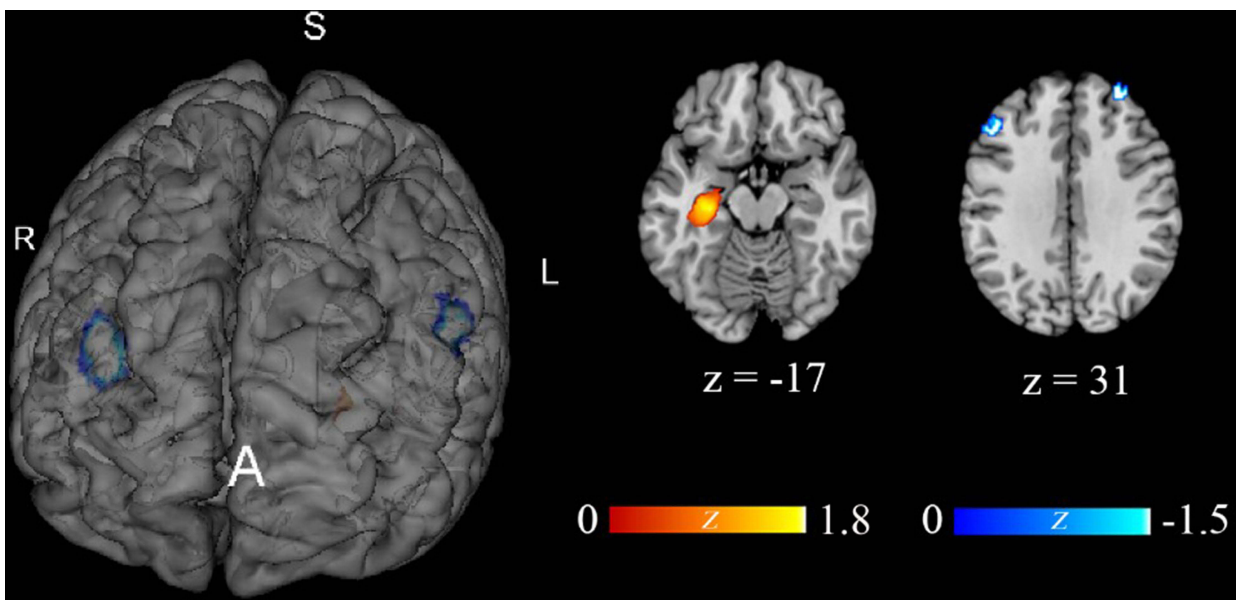

Figure 3 Effect of rs 1344706 risk allele on brain functional connectivity on the basis of rDLPFC seed region.

with neural activity only in the left anterior cingulate gyrus but not in rDLPFC, dorsomedial prefrontal cortex, left calcarine gyrus, and posterior cingulate cortex, which were reported in other studies. ${ }^{18,19,29}$ These differences may be due to a higher statistical powerful in our metaanalysis. Thus, relatively less regions were found to be associated with risk allele A. However, the total effect of this risk allele on brain function was never changed in our meta-analysis in healthy individuals.

The functional connectivity of the rDLPFC-left hippocampus in the rs1344706 risk allele carrier was significantly increased, which showed the positive and obviously strong effect of this gene in our meta-analysis. However, in patients with chronic schizophrenia, the hippocampalprefrontal functional connectivity seemed to be reduced in the resting state, suggesting the negative effect of rs1344706 risk allele. ${ }^{37}$ On the one hand, it may be due to differences in the observation indicators and clinical baselines of the included samples. On the other hand, it may be due to the difference in the races of subjects because the frequency of rs1344706 allele distribution varies widely among different populations, and this difference may directly affect the results of association studies.

Imaging genetic studies generally evaluate the risks of specific genes on functional development of the cerebrum. However, how the protein or nucleic acid produced by gene coding affects the execution of brain function is not clear. ZN804A is a schizophrenia susceptibility gene that is strongly supported by GWA analysis. In our meta-analysis and previous studies, rs1344706 risk allele influenced the different

Table 4 Summary of Functional Connectivity and Neural Activity Studies Not Included in AES-SDM Analysis

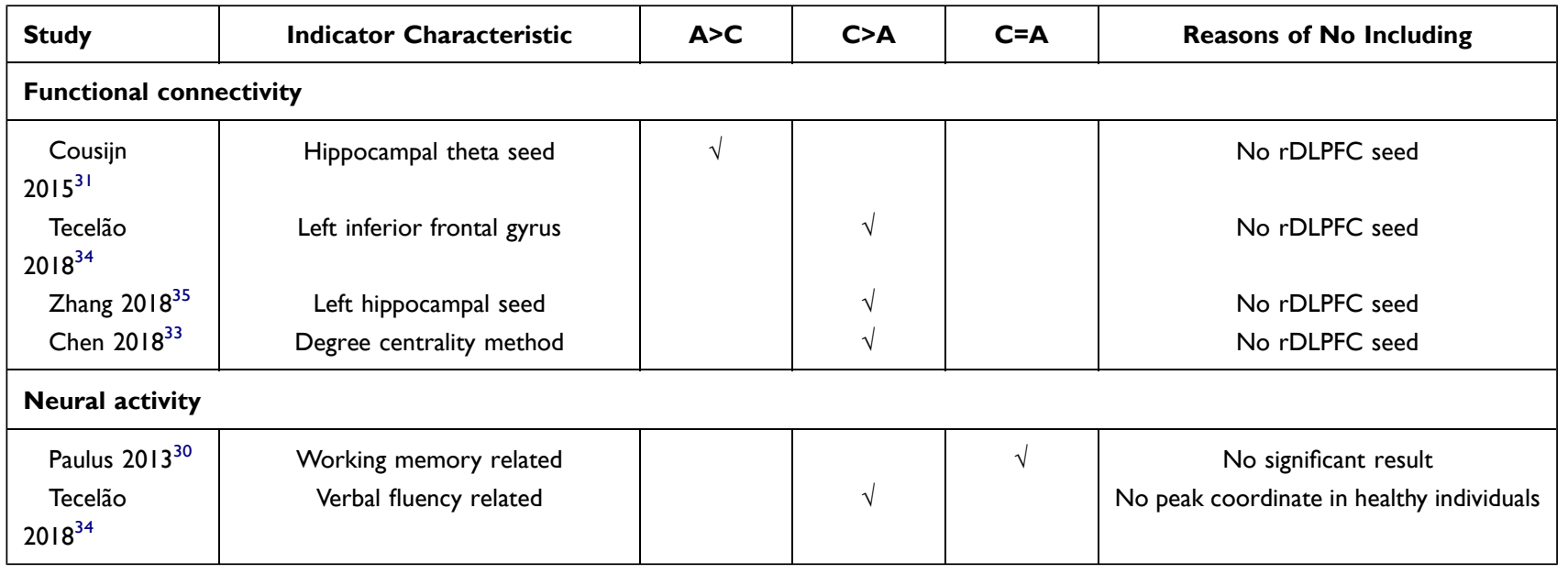

Notes: $A>C$ means risk allele had positive effect on functional connectivity or neural activity; $C>A$ means risk allele had negative effect on functional connectivity or neural activity; $C=A$ means risk allele had no significant effect on functional connectivity or neural activity.

Abbreviation: rDLPFC, right dorsolateral prefrontal cortex. 


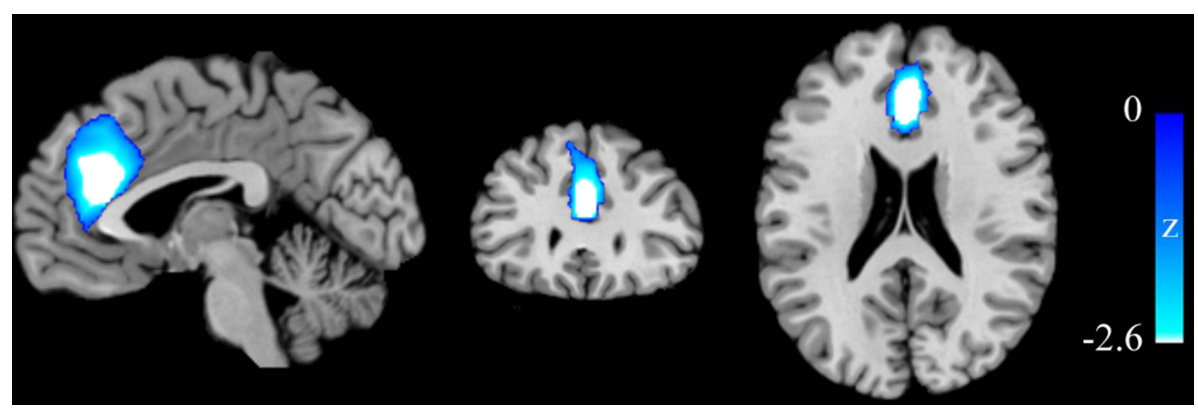

Figure 4 Effect of rs|344706 risk allele on neural activity.

regions of the brain. In complementary gene expression analyses, the rs1344706 risk allele was found to be associated with increased ZNF804A exonic transcription levels in the hippocampus, medulla oblongata, occipital cortex, and other regions. $^{34,38}$ Hence, the effects of the risk allele on brain function may result from the different expression of ZNF804A gene in different brain regions. The dosage of ZNF804A may determine some functions of the human brain. Animal experiments, biological information analysis, and functional MRI scanning suggested that the polymorphic site of rs1344706 affects human brain functional connectivity and is related to the development of white matter circuits. ${ }^{39}$
For the ZNF804A gene, studies have shown that the gene only affects the early development of the brain, but has no effect on the mature adult brain. ${ }^{40}$ However, from the perspective of autopsy, the ZNF804A gene showed differential expression between healthy individuals and patients with schizophrenia. ${ }^{41-43}$ These pieces of evidence indirectly or directly reveal that ZNF804A expression is associated with brain development and different phenotypes. Nevertheless, direct experimental data on the ZNF804A gene rs1344706 risk allele are still lacking to determine how brain development affects different individuals to exhibit different brain function characteristics.

Table 5 Leave One Out Jackknife Sensitivity Analysis Results

\begin{tabular}{|c|c|c|c|c|}
\hline \multirow{2}{*}{$\begin{array}{l}\text { Study of Leave } \\
\text { Out }\end{array}$} & \multicolumn{3}{|c|}{ Functional Connectivity } & \multirow{2}{*}{$\begin{array}{c}\text { Neural Activity } \\
\text { Left Anterior Cingulate } \\
\text { Gyrus }\end{array}$} \\
\hline & $\begin{array}{l}\text { rDLPFC-Left } \\
\text { Hippocampus }\end{array}$ & $\begin{array}{l}\text { rDLPFC-Left Middle } \\
\text { Frontal Gyrus }\end{array}$ & $\begin{array}{l}\text { rDLPFC- Right Middle } \\
\text { Frontal Gyrus }\end{array}$ & \\
\hline \multicolumn{5}{|c|}{ Functional connectivity } \\
\hline $\begin{array}{l}\text { Esslinger } \\
2009^{16}\end{array}$ & No significant changed & Peak coordinate changed & No significant changed & - \\
\hline $\begin{array}{l}\text { Esslinger } \\
2011^{26}\end{array}$ & No significant changed & No significant changed & No significant changed & - \\
\hline Rasetti $20 \mathrm{II}^{17}$ & No significant changed & Reported no region & Reported no region & - \\
\hline Walter $201 I^{18}$ & No significant changed & No significant changed & No significant changed & - \\
\hline Paulus $2013^{30}$ & No significant changed & No significant changed & No significant changed & - \\
\hline Thurin $2013^{27}$ & No significant changed & No significant changed & No significant changed & - \\
\hline Zhang $2016^{32}$ & No significant changed & No significant changed & No significant changed & - \\
\hline Zhao $2020^{36}$ & No significant changed & No significant changed & No significant changed & - \\
\hline \multicolumn{5}{|l|}{ Neural activity } \\
\hline Walter $2011^{18}$ & - & - & - & No significant changed \\
\hline Linden $2013^{28}$ & - & - & - & No significant changed \\
\hline Thurin $2013^{27}$ & - & - & - & Peak coordinate changed \\
\hline Mohnke & - & - & - & No significant changed \\
\hline $2014^{29}$ & & & & \\
\hline Cui $2019^{19}$ & - & - & - & No significant changed \\
\hline
\end{tabular}

Abbreviation: rDLPFC, right dorsolateral prefrontal cortex. 
Table 6 Sensitivity Analysis of Function Connections Based on rDLPFC Seed Region When Removed Two Studies

\begin{tabular}{|c|c|c|c|c|c|c|}
\hline \multirow[t]{2}{*}{ Connected Region } & \multicolumn{5}{|c|}{ Peak Coordinate } & \multirow[t]{2}{*}{ Cluster Size in Voxels } \\
\hline & \multicolumn{3}{|c|}{ MNI $(x, y, z)$} & SDM-z & $p$ & \\
\hline \multicolumn{7}{|c|}{ Positive effects of rs I344706 risk alleles } \\
\hline Left hippocampus & -28 & -20 & -14 & 1.763 & $<0.001$ & 578 \\
\hline \multicolumn{7}{|c|}{ Negative effects of rs I344706 risk alleles } \\
\hline Left middle frontal gyrus & -46 & 32 & 36 & -1.494 & $<0.001$ & 210 \\
\hline Right middle frontal gyrus & 28 & 50 & 30 & -1.342 & $<0.001$ & 167 \\
\hline
\end{tabular}

Abbreviation: rDLPFC, right dorsolateral prefrontal cortex.

ZNF804A is closely related to the cognitive function of the brain. ${ }^{44}$ An investigation in healthy subjects showed that cortical connectivity and activation were related to rs1344706 during performance on a theory of mind task (which measures the participant's ability to infer mental state). ${ }^{16}$ The regional activation of the temporoparietal cortex and rDLPFC, which are implicated in the function in theory of mind, was found to be affected by the dosage of risk allele. ${ }^{18}$ Our meta-analysis conducted in healthy individuals revealed the association between ZNF804A rs1344706 allele gene expression and rDLPFC seeded functional connectivity, highlighting the key role of
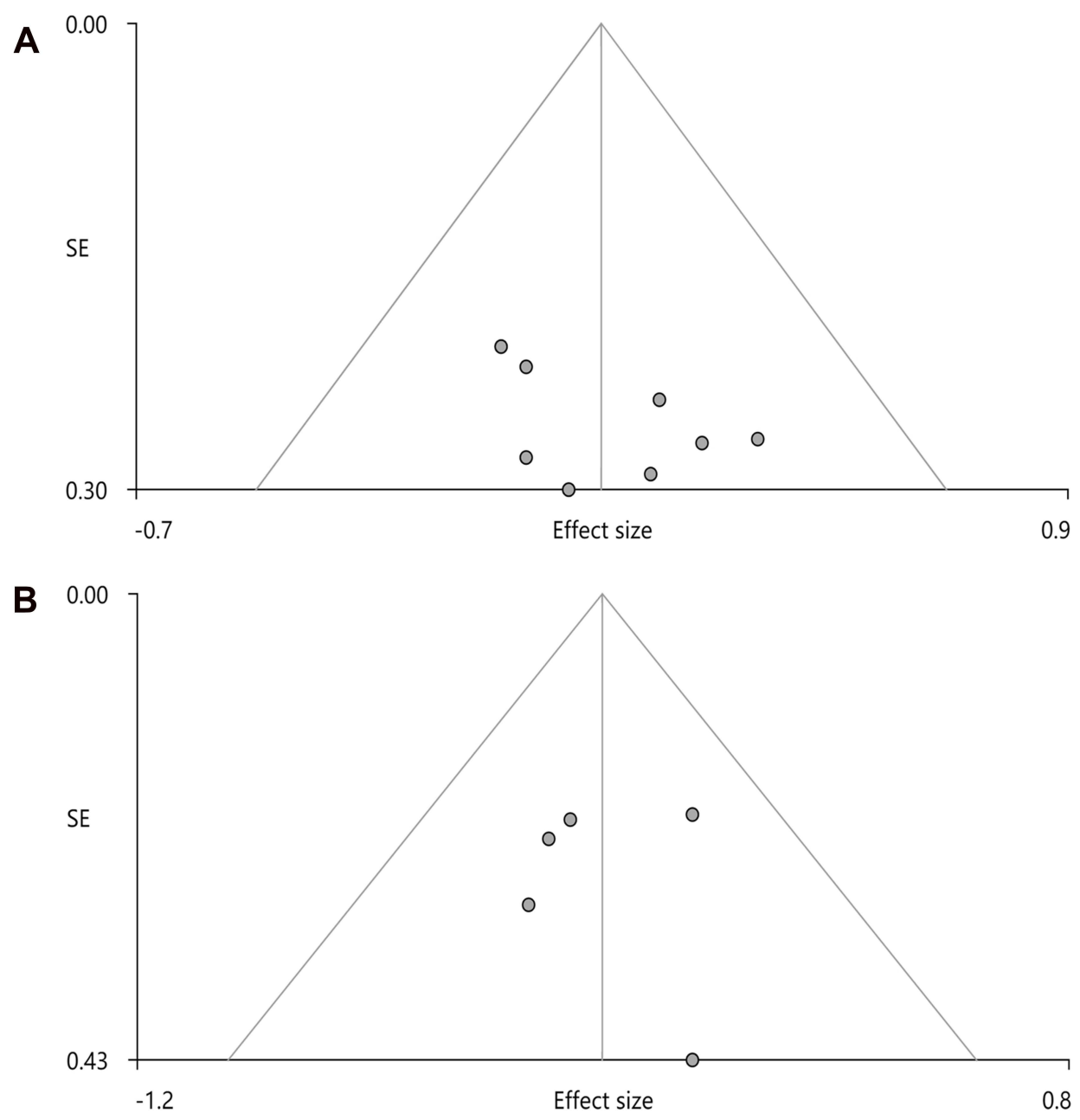

Figure 5 Publication bias of funnel plots.

Note: (A) Functional connectivity; (B) Neural activity. 
ZNF804A in cognitive function. One important characteristic of patients with schizophrenia is impaired cognitive function. Thus, the function of this risk gene in healthy individuals could also reflect the potential effect of rs1344706 on the abnormal cognitive function of patients with schizophrenia.

No publication bias was found in this meta-analysis, but a significant heterogeneity existed when synthesizing functional connectivity indicators. The heterogeneity most likely come from the different analytical indicators and scanning models among the including studies. In general, this study clarified the effect of ZNF804A gene rs1344706 risk allele on function through meta-analysis and provided a more in-depth reference for the study of genetic related pathogenesis of mental disorders such as schizophrenia.

\section{Conclusion}

The ZNF804A gene rs1344706 polymorphism has a strong correlation with brain functional connectivity and neural activity in healthy individuals. The risk allele A may be associated with abnormal or changed brain function in individuals and play a key role in the execution of brain function. This meta-analysis provides important information for the further study of genetic related mechanisms of schizophrenia and other mental disorders. The relationship between the rs1344706 risk allele of ZNF804A and brain dysfunction also provides an important reference for the prevention and treatment of mental disorders.

\section{Acknowledgments}

This study was supported by grants from the Natural Science Foundation of Chengdu Medical College (grant no. CYZ1808), Research Foundation of Sichuan Applied Psychology Research Center (grant no. CSXL-192A13), and Student Innovation and Entrepreneurship Training Program for Universities in Sichuan (grant no. S201913705056).

\section{Author Contributions}

All authors contributed to data analysis, drafting, and revision of the article. All authors have agreed on the journal to which the article will be submitted, gave final approval of the version to be published, and agree to be accountable for all aspects of the work.

\section{Disclosure}

The authors report no conflicts of interest in this work.

\section{References}

1. Lisman JE, Coyle JT, Green RW, et al. Circuit-based framework for understanding neurotransmitter and risk gene interactions in schizophrenia. Trends Neurosci. 2008;31(5):234-242. doi:10.1016/j. tins.2008.02.005

2. Steinberg S, Mors O, Borglum AD, et al. Expanding the range of ZNF804A variants conferring risk of psychosis. Mol Psychiatry. 2011;16(1):59-66. doi:10.1038/mp.2009.149

3. Tao R, Cousijn H, Jaffe AE, et al. Expression of ZNF804A in human brain and alterations in schizophrenia, bipolar disorder, and major depressive disorder: a novel transcript fetally regulated by the psychosis risk variant rs1344706. JAMA Psychiatry. 2014;71 (10):1112-1120. doi:10.1001/jamapsychiatry.2014.1079

4. Voineskos AN, Lerch JP, Felsky D, et al. The ZNF804A gene: characterization of a novel neural risk mechanism for the major psychoses. Neuropsychopharmacology. 2011;36(9):1871-1878. doi:10.1038/npp.2011.72

5. O'Donovan MC, Craddock N, Norton N, et al. Identification of loci associated with schizophrenia by genome-wide association and follow-up. Nat Genet. 2008;40(9):1053-1055. doi:10.1038/ng.201

6. Xiao B, Li W, Zhang H, et al. To the editor: association of ZNF804A polymorphisms with schizophrenia and antipsychotic drug efficacy in a Chinese Han population. Psychiatry Res. 2011;190(2-3):379-381. doi:10.1016/j.psychres.2011.05.031

7. Schwab SG, Kusumawardhani A, Dai N, et al. Association of rs1344706 in the ZNF804A gene with schizophrenia in a case/control sample from Indonesia. Schizophr Res. 2013;147(1):46-52. doi:10.1016/j.schres.2013.03.022

8. Wang S, Li W, Zhang H, Zhao J, Lyu L. The relationship between genetic polymorphism of ZNF804A and drug efficacy to schizophrenia: a meta-analysis. Chin J Behav Med Brain Sci. 2012;021 (10):897-899. doi:10.3760/cma.j.issn.1674-6554.2012.10.010

9. Wang D, Wang Y, Chen Y, et al. Ethnicity-dependent effects of Zinc finger $804 \mathrm{~A}$ variant on schizophrenia: a systematic review and meta-analysis. Psychiatr Genet. 2021;31(1):21-28. doi:10.1097/ YPG.0000000000000275

10. Lishman WA. The apparatus of mind: brain structure and function in mental disorder. Psychosomatics. 1983;24(8):699-703, 709-611, 714-620. doi:10.1016/S0033-3182(83)73166-X

11. Ragland JD, Ranganath C, Harms MP, et al. Functional and neuroanatomic specificity of episodic memory dysfunction in schizophrenia: a functional magnetic resonance imaging study of the relational and item-specific encoding task. JAMA Psychiatry. 2015;72(9):909-916. doi:10.1001/jamapsychiatry.2015.0276

12. Shad MU, Keshavan MS. Neurobiology of insight deficits in schizophrenia: an fMRI study. Schizophr Res. 2015;165(2-3):220-226. doi:10.1016/j.schres.2015.04.021

13. Walters JT, Corvin A, Owen MJ, et al. Psychosis susceptibility gene ZNF804A and cognitive performance in schizophrenia. Arch Gen Psychiatry. 2010;67(7):692-700. doi:10.1001/archgenpsychiatry.2010.81

14. Donohoe G, Rose E, Frodl T, et al. ZNF804A risk allele is associated with relatively intact gray matter volume in patients with schizophrenia. Neuroimage. 2011;54(3):2132-2137. doi:10.1016/j. neuroimage.2010.09.089

15. Lencz T, Szeszko PR, DeRosse P, et al. A schizophrenia risk gene, ZNF804A, influences neuroanatomical and neurocognitive phenotypes. Neuropsychopharmacology. 2010;35(11):2284-2291. doi:10.1038/npp.2010.102

16. Esslinger C, Walter H, Kirsch $P$, et al. Neural mechanisms of a genome-wide supported psychosis variant. Science. 2009;324 (5927):605. doi:10.1126/science.1167768

17. Rasetti R, Sambataro F, Chen Q, Callicott JH, Mattay VS. Altered cortical network dynamics: a potential intermediate phenotype for schizophrenia and association with ZNF804A. Arch Gen Psychiatry. 2011;68(12):1207. doi:10.1001/archgenpsychiatry.2011.103 
18. Walter H, Schnell K, Erk S, et al. Effects of a genome-wide supported psychosis risk variant on neural activation during a theory-ofmind task. Mol Psychiatry. 2011;16(4):462-470. doi:10.1038/ mp.2010.18

19. Cui L, Wang F, Chang M, et al. Spontaneous regional brain activity in healthy individuals is nonlinearly modulated by the interaction of ZNF804A rs1344706 and COMT rs4680 polymorphisms. Neurosci Bull. 2019;35(4):735-742. doi:10.1007/s12264-019-00357-w

20. Moher D, Shamseer L, Clarke M, et al. Preferred reporting items for systematic review and meta-analysis protocols (PRISMA-P) 2015 statement. Syst Rev. 2015;4(1):1. doi:10.1186/2046-4053-4-1

21. Wang S, He Y, Chen Z, Li Y, Zhao J, Lyu L. Pleiotropic action of genetic variation in ZNF804A on brain structure: a meta-analysis of magnetic resonance imaging studies. Neuropsychiatr Dis Treat. 2019;15:721-729. doi:10.2147/NDT.S174728

22. Radua J, Mataix-Cols D. Voxel-wise meta-analysis of grey matter changes in obsessive-compulsive disorder. Br J Psychiatry. 2009;195 (5):393-402. doi:10.1192/bjp.bp.108.055046

23. Xiao B, Wang S, Liu J, Meng T, He Y, Luo X. Abnormalities of localized connectivity in schizophrenia patients and their unaffected relatives: a meta-analysis of resting-state functional magnetic resonance imaging studies. Neuropsychiatr Dis Treat. 2017;13:467-475. doi:10.2147/ndt.S126678

24. Wise T, Radua J, Via E, et al. Common and distinct patterns of grey-matter volume alteration in major depression and bipolar disorder: evidence from voxel-based meta-analysis. Mol Psychiatry. 2017;22(10):1455-1463. doi:10.1038/mp.2016.72

25. Raina SK. Interpreting forest plots and funnel plots in meta-analysis. Neurol India. 2016;64(4):840. doi:10.4103/0028-3886.185366

26. Esslinger C, Kirsch P, Haddad L, et al. Cognitive state and connectivity effects of the genome-wide significant psychosis variant in ZNF804A. Neuroimage. 2011;54(3):2514-2523. doi:10.1016/j. neuroimage.2010.10.012

27. Thurin K, Rasetti R, Sambataro F, et al. Effects of ZNF804A on neurophysiologic measures of cognitive control. Mol Psychiatry. 2013;18(8):852-854. doi:10.1038/mp.2012.134

28. Linden DE, Lancaster TM, Wolf C, et al. ZNF804A genotype modulates neural activity during working memory for faces. Neuropsychobiology. 2013;67(2):84-92. doi:10.1159/000344001

29. Mohnke S, Erk S, Schnell K, et al. Further evidence for the impact of a genome-wide-supported psychosis risk variant in ZNF804A on the Theory of Mind Network. Neuropsychopharmacology. 2014;39 (5):1196-1205. doi:10.1038/npp.2013.321

30. Paulus FM, Krach S, Bedenbender J, et al. Partial support for ZNF804A genotype-dependent alterations in prefrontal connectivity. Hum Brain Mapp. 2013;34(2):304-313. doi:10.1002/hbm.21434

31. Cousijn H, Tunbridge EM, Rolinski M, et al. Modulation of hippocampal theta and hippocampal-prefrontal cortex function by a schizophrenia risk gene. Hum Brain Mapp. 2015;36 (6):2387-2395. doi:10.1002/hbm.22778

Neuropsychiatric Disease and Treatment

\section{Publish your work in this journal}

Neuropsychiatric Disease and Treatment is an international, peerreviewed journal of clinical therapeutics and pharmacology focusing on concise rapid reporting of clinical or pre-clinical studies on a range of neuropsychiatric and neurological disorders. This journal is indexed on PubMed Central, the 'PsycINFO' database and CAS, an
32. Zhang Z, Chen X, Yu P, et al. Effect of rs 1344706 in the ZNF804A gene on the connectivity between the hippocampal formation and posterior cingulate cortex. Schizophr Res. 2016;170(1):48-54. doi:10.1016/j.schres.2015.11.024

33. Chen X, Zhang Z, Zhang Q, et al. Effect of rs1344706 in the ZNF804A gene on the brain network. Neuroimage Clin. 2018;17:1000-1005. doi:10.1016/j.nicl.2017.12.017

34. Tecelão D, Mendes A, Martins D, et al. The impact of psychosis genome-wide associated ZNF804A variation on verbal fluency connectivity. $J$ Psychiatr Res. 2018;98:17-21. doi:10.1016/j. jpsychires.2017.12.005

35. Zhang Y, Yan H, Liao J, et al. ZNF804A variation may affect hippocampal-prefrontal resting-state functional connectivity in schizophrenic and healthy individuals. Neurosci Bull. 2018;34 (3):507-516. doi:10.1007/s12264-018-0221-y

36. Zhao W, Chen X, Zhang Q, et al. Effect of ZNF804A gene polymorphism (rs1344706) on the plasticity of the functional coupling between the right dorsolateral prefrontal cortex and the contralateral hippocampal formation. NeuroImage. 2020;27:102279. doi:10.1016/j. nicl.2020.102279

37. Rotarska-Jagiela A, van de Ven V, Oertel-Knochel V, Uhlhaas PJ, Vogeley K, Linden DE. Resting-state functional network correlates of psychotic symptoms in schizophrenia. Schizophr Res. 2010;117 (1):21-30. doi:10.1016/j.schres.2010.01.001

38. Ramasamy A, Trabzuni D, Guelfi S, et al. Genetic variability in the regulation of gene expression in ten regions of the human brain. Nat Neurosci. 2014;17(10):1418-1428. doi:10.1038/nn.3801

39. Meyer-Lindenberg A. Imaging genetics of schizophrenia. Dialogues Clin Neurosci. 2010;12(4):449-456. doi:10.31887/DCNS.2010.12.4/ amlindenberg

40. Hill MJ, Bray NJ. Evidence that schizophrenia risk variation in the ZNF804A gene exerts its effects during fetal brain development. Am J Psychiatry. 2012;169(12):1301-1308. doi:10.1176/appi. ajp.2012.11121845

41. Schultz CC, Nenadic I, Riley B, et al. ZNF804A and cortical structure in schizophrenia: in vivo and postmortem studies. Schizophr Bull. 2014;40(3):532-541. doi:10.1093/schbul/sbt123

42. Okada T, Hashimoto R, Yamamori H, et al. Expression analysis of a novel mRNA variant of the schizophrenia risk gene ZNF804A. Schizophr Res. 2012;141(2-3):277-278. doi:10.1016/j.schres.2012.08.015

43. Buonocore F, Hill MJ, Campbell CD, et al. Effects of cis-regulatory variation differ across regions of the adult human brain. Hum Mol Genet. 2010;19(22):4490-4496. doi:10.1093/hmg/ddq380

44. Chang H, Xiao X, Li M. The schizophrenia risk gene ZNF804A: clinical associations, biological mechanisms and neuronal functions. Mol Psychiatry. 2017;22(7):944-953. doi:10.1038/mp.2017.19 is the official journal of The International Neuropsychiatric Association (INA). The manuscript management system is completely online and includes a very quick and fair peer-review system, which is all easy to use. Visit http://www.dovepress.com/testimonials.php to read real quotes from published authors. 